\title{
Emergence of chaos and controlled photon transfer in a cavity-QED network
}

\author{
Amit Dey* and Manas Kulkarni ${ }^{\dagger}$ \\ International Centre for Theoretical Sciences, Tata Institute of Fundamental Research, Bengaluru, 560089, India
}

(Received 26 May 2020; accepted 23 September 2020; published 9 October 2020)

\begin{abstract}
We develop optimal protocols for efficient photon transfer in a cavity-QED network. This is executed through stimulated Raman adiabatic passage scheme where time-varying inductive or capacitive couplings (with carefully chosen sweep rate) play a key role. We work in a regime where the semiclassical limit is valid, and we investigate the dynamical chaos caused by the light-matter coupling. We show that this plays a crucial role in estimating the lower bound on the sweep rate for ensuring efficient photon transfer. We present Hermitian as well as an open quantum system extension of the model. Without loss of generality, we study the three cavity and four cavity cases and our results can be adapted to larger networks. Our analysis is also significant in designing transport protocols aimed for nonlinear open quantum systems, in general.
\end{abstract}

DOI: 10.1103/PhysRevResearch.2.042004

Introduction. High-precision controllability of cavity-QED (c-QED) systems and the potential of fabricating artificial lattices [1-4] highlights c-QED systems as an important component of quantum network [5-10]. The accessibility of a wide range of light-matter interaction (nonlinearity) signify its relevance for simulating strongly correlated systems [11-14] and demonstrate various phases, such as localization-delocalization [15-17], superfluid-Mott insulator $[11,13,18-21]$ phases. Interesting and important phenomena, such as qubit state preparation, photon-assisted transfer $[5,7,22,23]$, and various quantum correlation measures [23-25], to name a few, have also been recently investigated.

Population transport through a nonlinear network [such as multimode Bose-Hubbard (BH) systems] results in intricate physics of various types of instabilities [26,27]. Apart from energetic instability [27] due to nonlinear eigenstates (of the problem in the semiclassical limit), chaos can play major role in determining transfer efficiency [26]. Therefore, a judicious control of system parameters is crucial to tackle such sensitive physical processes. Nonlinear stimulated Raman adiabatic passage (STIRAP) consisting of interacting atomic Bose-Einstein (BEC) condensates has been analyzed semiclassically [26] as well as in a quantum many-body framework [28], and the role of various instabilities has been investigated theoretically. It has been shown that the adiabatic conditions for such processes get modified due to the emergence of chaos [26]. Another platform to investigate nonlinearity is a c-QED lattice. This platform precisely implements the Jaynes-Cummings nonlinearity which is very different from the $\mathrm{BH}$ nonlinearity in atomic BEC. In addition to this, a

\footnotetext{
*amit.dey.85@gmail.com

†manas.kulkarni@icts.res.in
}

Published by the American Physical Society under the terms of the Creative Commons Attribution 4.0 International license. Further distribution of this work must maintain attribution to the author(s) and the published article's title, journal citation, and DOI. dispersive regime of a c-QED can mimic the $\mathrm{BH}$ nonlinearity (Kerr type). Therefore, a c-QED lattice, being an efficient quantum simulator, demands an extensive analysis of nonlinear transport. Chaotic signature in systems where a single cavity is involved [29-33] has been investigated, and such systems can be considered to be a good testing bed for quantumclassical correspondence [30] of chaos. In a linear trimer of cavities [34], control of nondirected (unlike the STIRAP scheme) single-photon transfer is proposed by tuning the ratio of intercavity tunnelings in ultrastrong light-matter coupling regime. Although nonlinear contribution is studied for adiabatic light passage in terms of excitation power dependence [35], to the best of our knowledge, role of chaos in these optical processes remained elusive so far. The Jaynes-Cummings interaction-induced nonlinearity is exploited in coupled c-QED systems, and delocalized-localized phases have already been realized [15-17]. Furthermore, driven-dissipative preparation of exotic steady states in extended cavity systems paved the avenue of controlling photon propagation in scaledup architectures [15]. Therefore, a deeper understanding of aspects of nonlinear dynamics (such as efficient photon transfer) of these systems will significantly add to the existing control strategies, and it is much needed to open up myriad of technological applications [22]. Developing such protocols warrants a deep understanding of nonlinear systems and, subsequently, bringing in important notions (for, e.g., chaos) can play a paramount role in engineering the systems to ensure efficient transfer.

In this Rapid Communication, we investigate a c-QEDbased STIRAP and show that dynamical chaos sets the lower bound for the sweep rate (which quantifies how fast one tunes the coupling strength), resulting in efficient photon transfer. Without loss of generality, we study the case of three and four cavities, and by efficient photon transfer, we mean, a nearly $100 \%$, transfer of photons from the first cavity to the last cavity with almost no occupation of the intermediate cavities during the time evolution. Quantifying chaos by the Lyapunov exponent (LE) in the semiclassical limit, we make a connection with the sweep rate. This sets the 


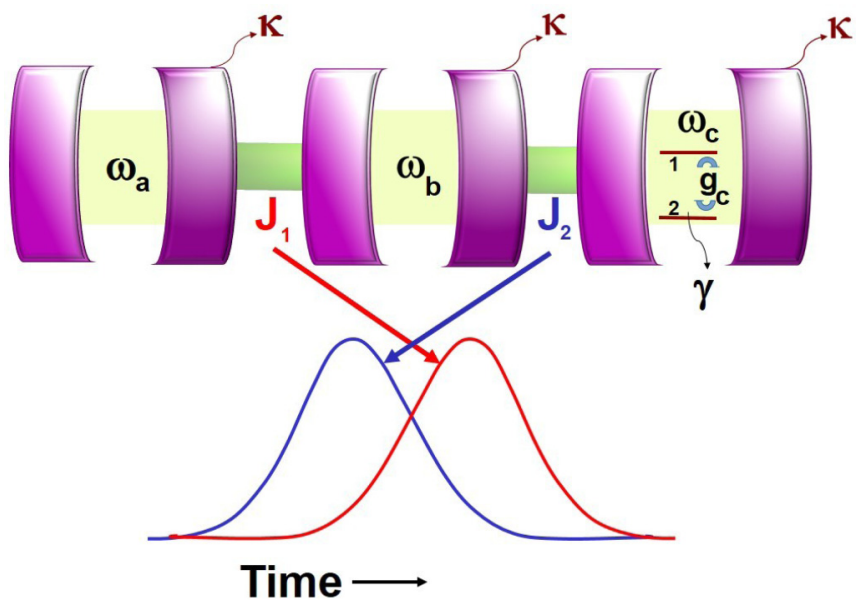

FIG. 1. Schematic presenting three coupled c-QEDs (with photon frequencies $\left.\omega_{a}, \omega_{b}, \omega_{c}\right)$ acting as a STIRAP. We consider cavity-qubit coupling $g_{c}$ only for cavity-c where the levels of the qubit are marked as 1 and 2. The objective of transferring photon population from cavity-a to cavity-c by negligibly populating the intermediate cavity-b is achieved through the counterintuitive sequence of Gaussian pulses for the tunneling $J_{1}, J_{2}$. Without loss of generality, we have shown the three-cavity case although our analysis holds for a large network.

lower bound on the sweep rate for the tuning parameters and thereby helps in achieving a strategy to ensure nearly $100 \%$ transfer of photons in an interacting/nonlinear system. Such a successful strategy for interacting systems lays a strong foundation for: (i) establishing photon-mediated communication by minimizing dissipation in a quantum network (because intermediate cavities are essentially empty in the process), and (ii) qubit-state transfer and its readout at the terminal cavity. Needless to mention, our model and analysis have broad applications in several physical platforms, such as cavityand circuit-QED-based systems, optomechanics, and hybrid quantum architectures, such as quantum-dot circuit-QED setups.

Starting from the model Hamiltonian, we work out the semiclassical equations of motion as we are in a regime where it is valid. We obtain the stationary point (SP) solutions for the Hermitian problem at every stage of the sweep (see the $J_{1}, J_{2}$ sweep in Fig. 1). These can be typically multivalued, but we track a special SP (SSP) branch that leads to a near-perfect transfer from the first to the last cavity with negligible content in the intermediate cavities. We present the STIRAP time dynamics for various sweep rates and analyze chaotic effects. For the SSP branch, we present the LE analysis at different sweep stages. This characterizes the chaotic aspects of the system. The results after including the inevitable presence of dissipation in experiments has also been discussed in the Supplemental Material [36]. Although much of our analysis relies on a semiclassical approximation, we have successfully demonstrated the consistency with an exact quantum calculation (see the Supplemental Material [36]). We, then, summarize our findings and discuss the future outlook.

Model and dynamical equations. The c-QED STIRAP given by the schematic Fig. 1 can be described by the time-dependent Hamiltonian given by

$$
\hat{H}=\sum_{j \in\{a, b, c\}} H_{j}-J_{1}(t)\left(\hat{a}^{\dagger} \hat{b}+\text { H.c. }\right)-J_{2}(t)\left(\hat{b}^{\dagger} \hat{c}+\text { H.c. }\right)
$$

where $\hat{H}_{a}=\omega_{a} \hat{a}^{\dagger} \hat{a}+\Omega_{a} \hat{s}_{a}^{z}+g_{a}\left(\hat{a}^{\dagger} \hat{s}_{a}^{-}+\right.$H.c. $)$describes the Jaynes-Cummings Hamiltonian for the cavity labeled by a (cavity-a). $\hat{a}$ destroys a photon with frequency $\omega_{a}$ in cavitya, $g_{a}$ denotes the light-matter (photon-qubit) interaction in cavity-a. We define $\hat{n}_{a, b, c}$ as the photon number operator in cavity-a, cavity-b, and cavity-c, respectively. The twolevel system with transition frequency $\Omega_{a, b, c}$ is described by the spin operators $\hat{s}_{a}^{\alpha}$ (where $\alpha \equiv\{x, y, z\}$ ). The timedependent couplings are defined as Gaussian pulses $J_{1,2}(t)=$ $K e^{-\left[\left(t-t_{1,2}\right) / \tau\right]^{2}}$ (with the sequence $t_{1}>t_{2}$ ), where $\tau$ is the pulse width and, therefore, $1 / \tau$ is the sweep rate (measured, henceforth, in units of $K$ ). Therefore, the Hamiltonian [Eq. (1)] is explicitly time dependent in a rescaled time $\tilde{t} \equiv t / \tau$. Throughout the Rapid Communication, we use $t_{1} / \tau=3.697, t_{2} / \tau=$ 2.4242 , and $K=1$. Our scheme can also be realized for other values of $t_{1,2}$, provided that $t_{1}>t_{2}$ and the $J_{1,2}$ have considerable overlap. The evolution under the time-dependent Hamiltonian [Eq. (1)] depends on the sweep rate of $J_{1,2}(t)$. The STIRAP scheme can be implemented, for, e.g., in coupled optical waveguides through variation of the spacings between the central and terminal waveguides [35,37-41]. A time-dependent inductive coupling can, in principle, be implemented by a SQUID-mediated tunable coupler $[42,43]$. Although there is no nonlinear photon-photon interaction in the Hamiltonian, perturbative treatment in the dispersive regime shows that the light-matter interaction induces such interaction [44]. However, we deal with a resonant situation $\left(\omega_{a, b, c}=\Omega_{a, b, c}\right)$ where the effect of anharmonicity is most manifest.

For a linear system (with $g_{a, b, c}=0$ ), Eq. (1) is just a standard STIRAP Hamiltonian, which ensures the existence of eigenstate $\left|\Psi_{0}\right\rangle \equiv \cos \Theta|A\rangle-\sin \Theta|C\rangle$ where $\cos \Theta=$ $\frac{J_{2}}{\sqrt{J_{1}^{2}+J_{2}^{2}}}$. Here, $|A\rangle(|C\rangle)$ is the state vector when the total population resides at cavity-a (cavity-c). This particular eigenstate does not project on to $|B\rangle$ and acts as a "dark state" [45-47], i.e., $\left\langle B \mid \Psi_{0}\right\rangle=0$. However, as $\Theta$ varies from 0 to $\pi / 2$ (i.e., a complete sweep of $J_{1,2}$, see Fig. 1), the state vector $\left|\Psi_{0}\right\rangle$ rotates from $|A\rangle$ to $|C\rangle$ resulting population transfer. We define transfer efficiency as $T=\frac{\left\langle\hat{n}_{c}(t \rightarrow \infty)\right\rangle}{\left\langle\hat{n}_{a}(t=0)\right\rangle}$. A semiclassical analog of an eigenstate is a solution of semiclassical equations of motion that do not evolve (i.e., a SP solution). Since, we will work in a regime where semiclassical approximation is valid, the analog of the dark state mentioned above is a SSP branch that leads to a perfect transfer from cavity-a to cavity-c (with zero content in cavity-b throughout the time dynamics). Therefore, for a successful adiabatic passage, we sweep the couplings $J_{1}, J_{2}$ slow enough (i.e., $1 / \tau$ is relatively small) so that the time evolution of the quantities follows the SSP branch.

In this Rapid Communication, we deal with a more complicated nonlinear situation and seek a similar photon transfer mechanism. In the large photon number limit, we exploit the approximation $\left\langle\hat{a} \hat{s}_{a}^{+}\right\rangle \approx\langle\hat{a}\rangle\left\langle\hat{s}_{a}^{+}\right\rangle$and treat the problem in a semiclassical framework $[17,48]$. For brevity, we use the 
following notations: $\langle\hat{a}\rangle \rightarrow a,\left\langle\hat{s}_{a}^{-}\right\rangle \rightarrow s_{a}$, and $\left\langle\hat{s}_{a}^{z}\right\rangle \rightarrow s_{a}^{z}$. Using the Heisenberg equation of motion and incorporating the above approximation, we obtain the dynamical equations given by the following five equations,

$$
\begin{gathered}
\dot{a}=i J_{1} b, \quad \dot{b}=-i \Delta b+i\left[J_{1} a+J_{2} c\right], \\
\dot{c}=-i g_{c} s_{c}+i J_{2} b, \\
\dot{s}_{c}=2 i g_{c} c s_{c}^{z}, \quad \dot{s}_{c}^{z}=-i g_{c}\left[s_{c}^{*} c-c^{*} s_{c}\right] .
\end{gathered}
$$

In obtaining Eqs. (2)-(4), we consider a setup where only third cavity has qubit-cavity coupling, i.e., $g_{a}=g_{b}=$ $0, g_{c} \neq 0$. This is because we want $\left\{n_{a}, n_{b}, n_{c}, s_{c}, s_{c}^{z}\right\} \equiv$ $\{N, 0,0,0,-0.5\}$ to be a SP at $\tilde{t}=0$ which is experimentally more feasible. The more general case, $g_{a, b, c} \neq 0$ will have more complicated SP solutions which, at $\tilde{t}=0$, may not have easily experimentally implementable initial conditions. In addition, we consider $\omega_{a}=\omega_{c}=\omega_{b}-\Delta$ without loss of generality and write Eqs. (2)-(4) in the rotating frame of frequency $\omega_{a, c}$. Here, $\Delta$ is the detuning of cavity-b from cavity-a/cavity-c and has a value $\Delta=0.5 \mathrm{~K}$ throughout the Rapid Communication. We work with the Hermitian problem governed by Hamiltonian [Eq. (1)], subjected to the conservation $n_{a}+n_{b}+n_{c}+s_{c}^{z}+1 / 2=N$. We show results pertaining to non-Hermitian processes in the Supplemental Material [36]. It turns out that, the non-Hermitian results for a complete sweep reflect similar features as the Hermitian case, provided the dissipation rates are considerably less than $1 / \tau$.

Stationary point solutions. The SP solutions are parametrized by $\tilde{t}$ and are, therefore, independent of $\tau$. To obtain SP solutions at various $\tilde{t}$ 's, we define the quantity $\hat{h} \equiv \hat{H}(\tilde{t})-\mu(\tilde{t})\left(\hat{n}_{a}+\hat{n}_{b}+\hat{n}_{c}+\hat{s}_{c}^{z}+1 / 2\right)$ where $\mu(\tilde{t})$ is a Lagrange multiplier (chemical potential) ensuring conservation. As in the previous section, we derive Heisenberg equations of motion with respect to $\hat{h}$ and by setting the time derivatives to zero, we write below four equations,

$$
\begin{gathered}
J_{1} b+\mu a=0, \quad \Delta b-J_{1} a-J_{2} c-\mu b=0, \\
J_{2} b-g_{c} s_{c}+\mu c=0, \quad 2 g_{c} c s_{c}^{z}+\mu s_{c}=0 .
\end{gathered}
$$

The above four equations along with the constraint $n_{a}+$ $n_{b}+n_{c}+s_{c}^{z}+1 / 2=N$ and the fact that $\frac{s_{c}^{2}+s_{c}^{*^{2}}}{2}+s_{c}^{z^{2}}=1 / 4$ (spin length) gives us six equations and six unknowns $\left(a, b, c, s_{c}, s_{c}^{z}, \mu\right)$. This has been solved numerically to yield SP solutions at respective $\tilde{t}$ values (Fig. 2). In particular, we look for SSP branch that facilitates cavity-a to cavity-c transfer by sweeping $\Theta(\tilde{t})$ from 0 to $\pi / 2$.

Numerical results. In this section, we show the SP solutions for $g_{c}=0.2 \mathrm{~K}$ and present the time dynamics for various sweep rates $1 / \tau$ (Fig. 2). We present SP solutions only for $n_{a}^{\mathrm{SP}}$ and $n_{c}^{\mathrm{SP}}$ since we are interested in the photon number of source (cavity-a) and terminal cavity (cavity-c). Among the various SP branches, there is a SSP branch that starts as $\left\{n_{a}^{\mathrm{SP}}=N=\right.$ $\left.20, n_{c}^{\mathrm{SP}}=0\right\}$ at $\tilde{t}=0$ and ends as $\left\{n_{a}^{\mathrm{SP}} \approx 0, n_{c}^{\mathrm{SP}} \approx N\right\}$ with a negligible value of $n_{b}^{\mathrm{SP}}$. This special branch is the nonlinear analog of the dark state. This SSP branch helps us to choose the correct initial conditions $\left\{a(0), b(0), c(0), s_{c}(0), s_{c}^{z}(0)\right\}$ that need to be subjected to Eqs. (2)-(4) for a chosen value
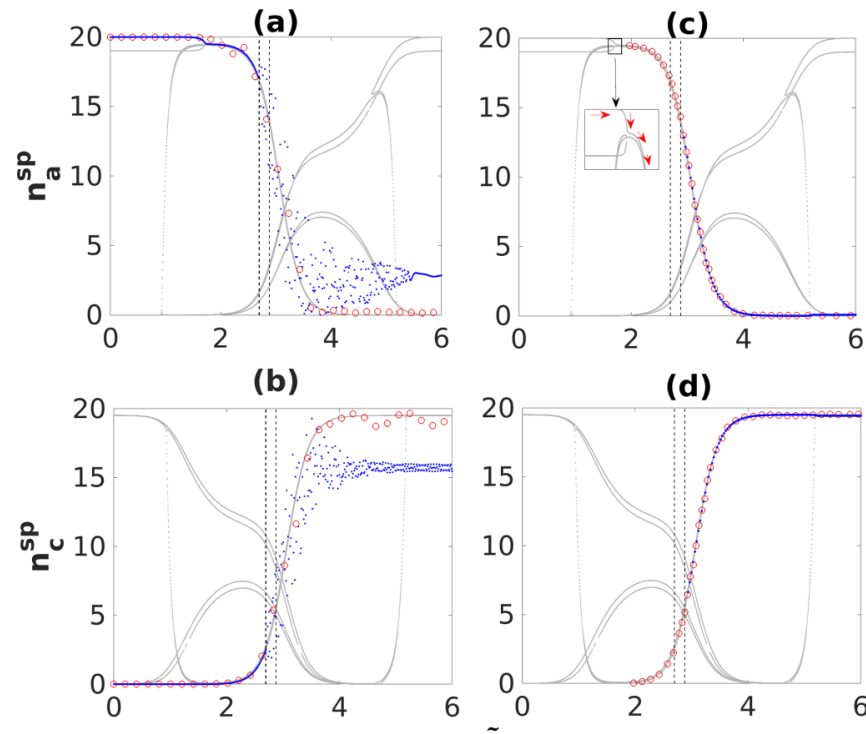

(d)

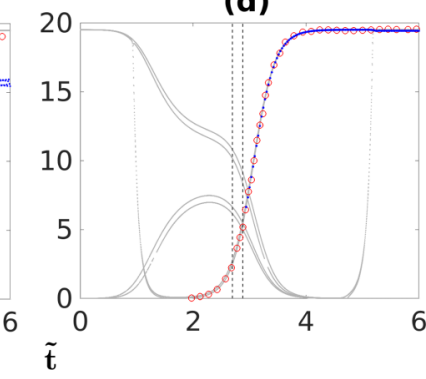

FIG. 2. The SP solutions for $g_{c}=0.2 \mathrm{~K}$ are presented by gray lines in all four panels. Red circles and blue dots give the real-time dynamics of cavity populations (under the time-dependent Hamiltonian in Eq. (1) at sweep rates $1 / \tau=0.0202$ (faster) and $1.2121 \times 10^{-4}$ (slower), respectively. The left and right vertical lines (at a certain $\tilde{t}_{\text {left }}$ and $\tilde{t}_{\text {right }}$ ) are predicted by the LE analysis of Figs. 3 and 4 (to be discussed later). These vertical lines give us the window of parameters $\left(\tilde{t}_{\text {left }}<\tilde{t}<\tilde{t}_{\text {right }}\right)$ of the Hamiltonian where the system is chaotic. (a) and (b), respectively, are cavity-a and cavity-c populations when the system is initialized at the SP solution at $\tilde{t}=0$ (i.e., $\left.\left\{n_{a}, n_{b}, n_{c}, s_{c}, s_{c}^{z}\right\} \equiv\{20,0,0,0,-0.5\}\right)$. On the other hand, in (c) and (d), we present two cases both in which we initiate the system at $\tilde{t}>0$ on the SSP branch. One case is in which the system is initiated immediately after the location of the inset in (c) (i.e., red circle, initiated at $\tilde{t}=1.9697$ with SSP solution $\left\{n_{a}, n_{b}, n_{c}, s_{c}, s_{c}^{z}\right\} \equiv$ $\{19.4542,0.0150,0.0396,0.4999,-0.0088\})$ and swept at a faster rate. Another case is when the system is initiated immediately after the chaotic window (i.e., blue dots, initiated at $\tilde{t}=2.9394$ with SSP $\left.\left\{n_{a}, n_{b}, n_{c}, s_{c}, s_{c}^{z}\right\} \equiv\{12.8592,0.0073,6.6399,0.4999,-0.0065\}\right)$ and swept at a slower rate. The inset in (c) shows the regime where SSP branch (marked by red arrows) shows a sharp change in SP solutions.

of pulse width $(\tau)$ in $J_{1}(t)$ and $J_{2}(t)$. In addition, we need to make sure that the time dynamics remains on the SSP branch. This can be achieved by choosing optimal (elaborated below) sweep rate $1 / \tau$, subsequently, leading to efficient photon transfer.

Figure 2 captures two main aspects. One is the SP solutions which are dependent only on $\tilde{t}$. The other aspect is the realtime dynamics (which depends on sweep rate $1 / \tau$ ) where one wishes it to adiabatically follow the SSP branch that leads to efficient photon transfer. It is worth recapping the adiabatic theorem which states that, upon slowly varying the parameters of the Hamiltonian, the system remains in the Hamiltonian's instantaneous eigenstate. We adapt a similar intuition for its semiclassical limit which forms the basis for the standard STIRAP scheme [46]. This implies that sweep rates cannot be too fast irrespective of whether the Hamiltonian is interacting or noninteracting. On the other hand, the sweep rates cannot 
be too slow if the system is interacting (chaotic to be more precise) as demonstrated in Fig. 2 and the caption therein. Figures 2(a) and 2(b) demonstrate near-perfect transfer when the couplings are swept relatively faster (red circles). A relatively slow sweep (blue dots) shows smooth following of the SSP branch only until the onset of chaos. Our findings, therefore, demonstrate that the standard notion of adiabaticity is contradicted when the system is chaotic [26]. To ensure a smooth following of the SSP branch even in the chaotic window, the sweep rate should be sufficiently faster. To show that chaos is the only origin of this breakdown, we show in Figs. 2(c) and 2(d) that, if the system is initiated by avoiding the chaotic region (shown as blue dots, slower sweep), then, it follows the SSP branch. Therefore, the standard notion of adiabaticity holds here. In the inset region of Fig. 2(c), one can note a sharp change in the SP solutions. To follow the SSP branch in this region the sweep rate needs to be slower than the rate of change in the corresponding energy of the SSP solution with respect to $\tilde{t}$. This is the reason why we have small deviations from SSP branch in Fig. 2(a) for the faster sweep rate case (red circles). The same faster sweep gives a smooth following of the SSP branch [Figs. 2(c) and 2(d)] if we initiate the system after the inset region. We have also performed analysis for higher $g_{c}$ values and our findings still hold (see the Supplemental Material [36]).

Lyapunov exponent analysis. In this section, we will perform a LE analysis that will quantify chaos, and this will turn out to be an important ingredient in executing the STIRAP scheme explained earlier. One starts with infinitesimally separated initial conditions and extracts LE from diverging copies of trajectories. Due to the fact that $n_{a}+n_{b}+n_{c}+s_{c}^{z}+1 / 2=$ $N$, our phase space is bounded. Consequently, the distance between the trajectories does not grow monotonically, and, at long times, this may produce a false estimate of LE. To circumvent this issue, we exploit the prescription of resetting the phase-space distance between trajectories in Refs. [49-53]. The method is described as follows:

Two phase-space trajectories are chosen so that they initially differ by phase-space distance $\delta_{0}$. The trajectories are allowed to diverge for a time-step $\xi$, and the new distance between the trajectories $\delta_{1}$ is reset to $\delta_{0}$. This procedure is repeated $M$ times where $M$ is large. The LE is then defined as $\lambda_{M}=\lim _{\delta_{0} \rightarrow 0} \frac{1}{M K \xi} \sum_{j=1}^{M} \log \left(\frac{\delta_{j}}{\delta_{0}}\right)$ where $\lambda_{M}$ means that we have computed the Lyapunov exponent up to the time $t=M \xi$. Here, $\delta_{j}$ denotes the deviation between the trajectories before the $j$ th reset. The maximum LE $\left(\lambda_{\max }\right)$ can be obtained by taking the limit $M \rightarrow \infty$ and a positive (zero) $\lambda_{\max }$ indicates a chaotic (nonchaotic) behavior. Resetting at every step ensures that the deviation of trajectories is well within the phase-space boundary.

In Fig. 3, in the left column, we plot $\lambda_{M}$ as a function of dimensionless time $K M \xi$. Each of the figure is for a representative $\tilde{t}$ value. It is to be noted that specifying a $\tilde{t}$, fixes the parameters of the Hamiltonian $J_{1}, J_{2}$, therefore, making the Hamiltonian explicitly time independent. The procedure we employ to generate Figs. 3(a)-3(c) is the following: We create two infinitesimally separated copies, $A, B$ such that $P^{(A)}=P^{\mathrm{SP}}(\tilde{t})$ and $P^{(B)}=P^{\mathrm{SP}}(\tilde{t})+\delta P(t)$ where $P$ denotes the set $\left\{a, b, c, s_{c}, s_{c}^{z}\right\}$. It is worth reemphasizing that $H(\tilde{t})$ is explicitly time independent for a particular $\tilde{t}$. As can be seen
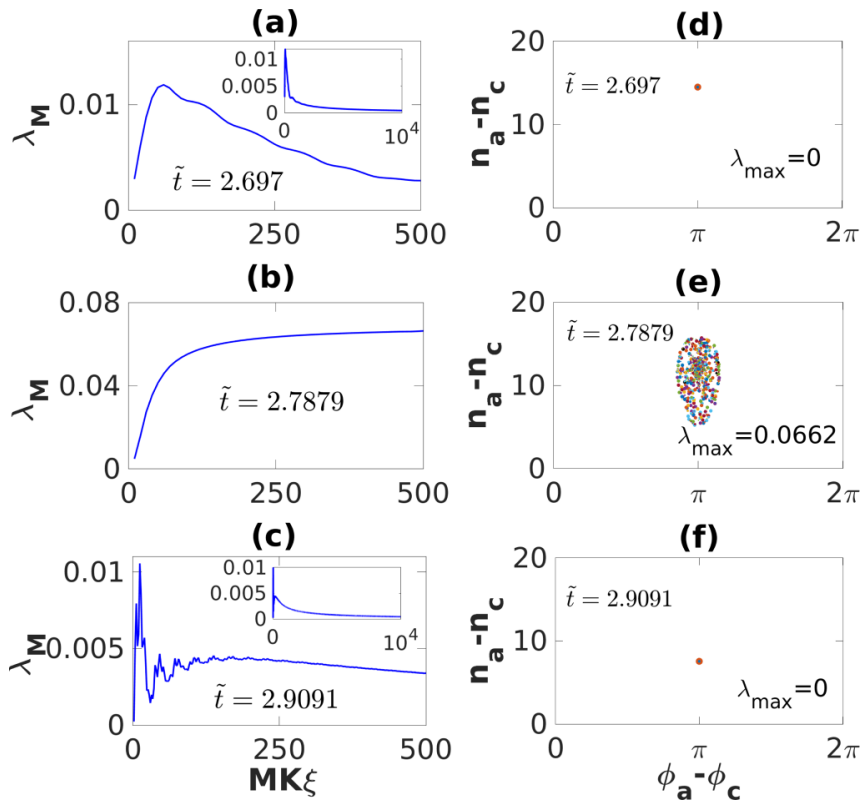

FIG. 3. (Left column) Lyapunov exponents for $g_{c}=0.2 \mathrm{~K}$. The insets in (a) and (c) demonstrate the vanishing of LE at sufficiently long times as one would expect. (Right column) The dynamics of an ensemble of phase space trajectories. An ensemble of ten samples is initiated around the SSP at that particular $\tilde{t}$ and evolved under timeindependent Hamiltonian $H(\tilde{t})$ for a long time. As shown clearly, whenever the Lyapunov exponent remains positive [see (b)], the phase space points spread out [see (e)] of the SSP demonstrating the chaos.

in Fig. 3 (left column), we see nonchaotic [Fig. 3(a)], chaotic [Fig. 3(b)], and again a nonchaotic [Fig. 3(c)] behavior. This is also reflected in the spreading features of phase-space points (right column of Fig. 3). Keeping in mind that we are interested in a transfer of photons from cavity-a to cavity-c, as a section of our phase space, we choose $n_{a}-n_{c}$ on the $y$ axis and the conjugate variable $\phi_{a}-\phi_{c}$ on the $x$ axis for the right column of Fig. 3. Figure 3(e) suggests that such chaotic stages should be crossed quickly (by choosing a sufficiently fast sweep rate) to minimize the effect of chaos. For higher $g_{c}$, the analog of Fig. 3 shows higher Lyapunov exponents and more prominent phase-space spreading (see the Supplemental Material [36]).

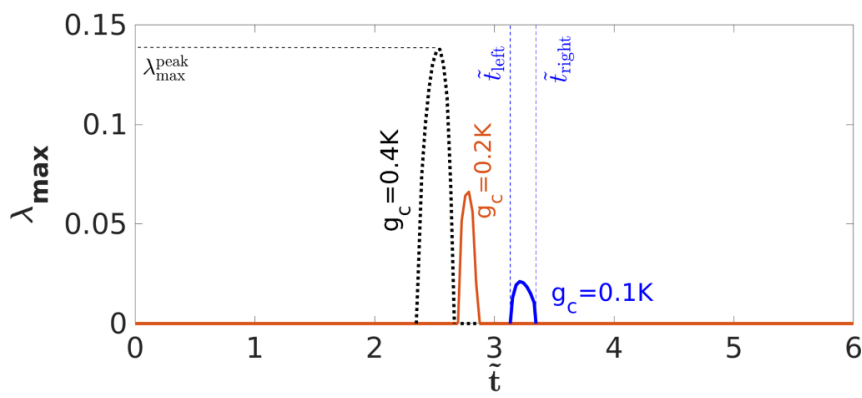

FIG. 4. $\lambda_{\max }$ is plotted with respect to $\tilde{t}$ for $g_{c}=0.1 \mathrm{~K}$ (solid blue or dark), $g_{c}=0.2 \mathrm{~K}$ (solid orange or gray), and $g_{c}=0.4 \mathrm{~K}$ (dotted black). Stronger light-matter coupling implies a larger $\lambda_{\max }^{\text {peak }}$. 

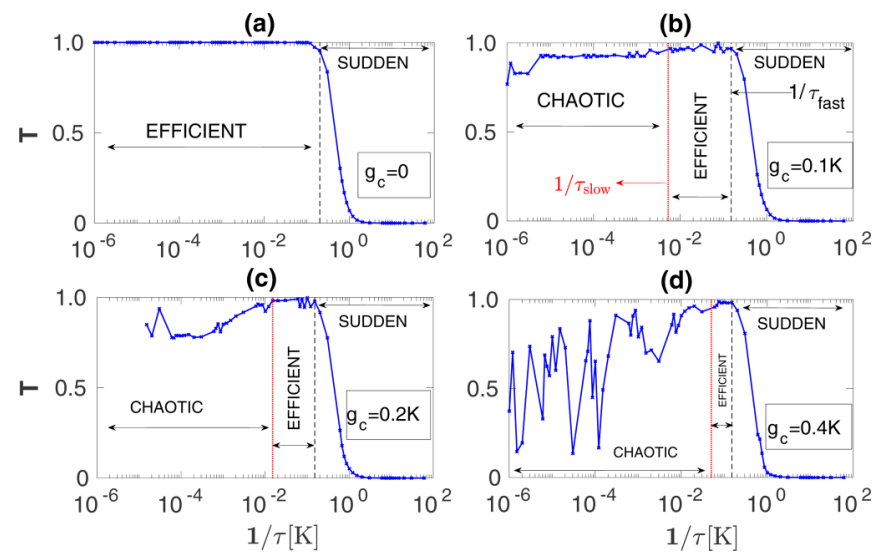

FIG. 5. $T$ vs the $1 / \tau$ plot. The black vertical line marks the standard adiabaticity requirement (fastest sweep rate possible) to achieve $95 \%$ efficiency. The red vertical dotted line represents the slowest sweep rate possible so as to assure, at least, $95 \%$ efficiency.

In Fig. 4 , we show the maximum LE $\left(\lambda_{\max }\right)$ as a function of $\tilde{t}$ for three values of $g_{c}$. As can be seen, for each $g_{c}$, there is a window $\tilde{t}_{\text {left }}<\tilde{t}<\tilde{t}_{\text {right }}$ where $\lambda_{\max }>0$. Therefore, $\mathrm{LE}$ analysis plays a paramount role in obtaining a window of $\tilde{t}$ where the system is chaotic. In particular, for the case of $g_{c}=$ $0.2 \mathrm{~K}$, the vertical lines in Fig. 2 are obtained by the above analysis.

Transfer efficiency. In Fig. 2, we showed that the dynamics and the transfer efficiency have strong dependence on the sweep rate. In Fig. 5, we plot the transfer efficiency $T$ with respect to $1 / \tau$ for various $g_{c}$ 's. Fig. 5(a) demonstrates that the slow sweep boundary does not exist for the noninteracting case, implying no presence of chaos. However, the sweep must not be too fast which will result in a violation of adiabaticity. This feature is reflected in the low-transfer sudden region beyond the black dashed lines in Fig. 5. The black dashed lines $\left(1 / \tau_{\text {fast }}\right)$ in Fig. 5 are constructed such that $T$ becomes less than 0.95 beyond the line. It is to be noted that $95 \%$ is generally regarded as satisfactory high efficiency [46]. In Figs. 5(b)-5(d), we show the interacting case $\left(g_{c} \neq 0\right)$. Compared to the noninteracting case [Fig. 5(a)], an additional chaos-dominated region emerges for the interacting case $\left(g_{c} \neq 0\right)$. The dotted red vertical line $\left(1 / \tau_{\text {slow }}\right)$ sets a lower bound on the sweep rate below which $T<0.95$. In other words, the sweep rate for high efficiency needs to satisfy $1 / \tau_{\text {fast }}>1 / \tau>1 / \tau_{\text {slow }}$. It is to be noted that, during real-time dynamics, the time spent within the chaotic window $\left(\tilde{t}_{\text {right }}-\tilde{t}_{\text {left }}\right) \tau$ is finite. This means that the relevant LE for chaotic spreading is the finite time LE (see Fig. 3). This automatically implies $1 / \tau_{\text {slow }}<\lambda_{\max }^{\text {peak }}$ where $\lambda_{\text {max }}^{\text {peak }}$ is the peak of $\lambda_{\max }$ for a given $g_{c}$ (see Fig. 4). Therefore, this finding relates LE to the lower bound on sweep rate. For higher $g_{c}, 1 / \tau_{\text {slow }}$ increases thereby shrinking the efficient region and widening the chaotic regime.

Four-cavity STIRAP scheme. To demonstrate scalability, we extend the three-cavity network to a four-cavity network (cavity-a, cavity-b, cavity-c, and cavity-d) where cavity-d houses a qubit. We couple the cavities by counterintuitive tunneling sequence shown in the inset of Fig. 6(a). Figure 6(a) shows near-unity transfer from cavity-a to cavity-d for a lin-
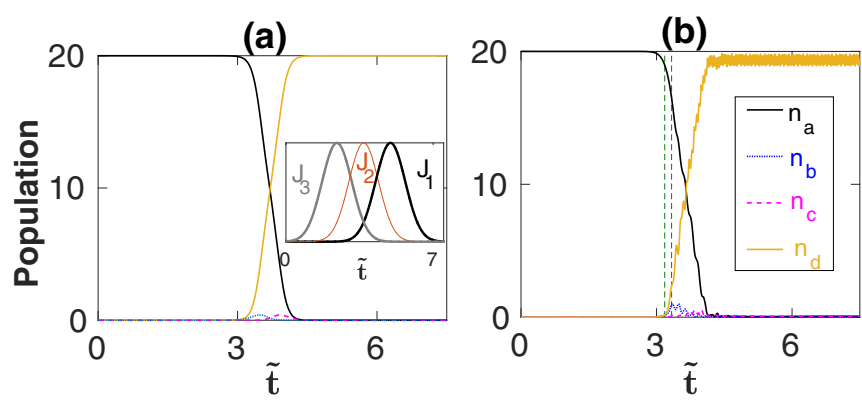

FIG. 6. Population dynamics for a four-cavity network with counterintuitive pulse sequence shown in the inset of (a). Dynamics of $n_{a}$ (black solid), $n_{b}$ (dotted blue), $n_{c}$ (dashed magenta), and $n_{d}$ [solid yellow (gray)] are shown for (a) linear $\left(g_{a, b, c, d}=0\right)$ case with $1 / \tau=0.00379$ and (b) nonlinear $\left(g_{a, b, c}=0, g_{d}=0.2 \mathrm{~K}\right)$ network with $1 / \tau=0.0101$. The dashed vertical lines in (b) marks the chaotic window whose potentially detrimental effect (for successful photon transfer) can be overcome with a faster sweep. For both (a) and (b), the system is initialized at the SSP solution of the four-cavity network at $\tilde{t}=0$ (i.e., $\left\{n_{a}, n_{b}, n_{c}, n_{d}, s_{d}, s_{d}^{z}\right\} \equiv\{20,0,0,0,0,-0.5\}$ ).

ear closed-system case. Figure 6(b) demonstrates near-perfect efficiency for the nonlinear case $\left(g_{d} \neq 0\right)$ when the parameters are swept at a faster rate. For a slower sweep rate (not shown here), similar to the three-cavity case [blue dots in Figs. 2(a) and 2(b)], we find that the efficiency is hindered by chaos.

Conclusions and outlook. We have demonstrated a protocol for achieving high transfer efficiency in an interacting c-QED STIRAP network. Such protocols are far from obvious given the fact that we are dealing with a scalable interacting system. We have found and exploited the deep connection between LE and nonlinear STIRAP schemes. Although, our protocols are developed on a semiclassical platform, we show that the resulting optimal choice of parameters successfully achieve our target for the fully quantum cases considering the Fock state, coherent state, and cat state as the initial state (see the Supplemental Material [36]). A further interesting direction could be a rigorous analysis of Linblad master equations [54] for our STIRAP setup. Needless to mention, analytical results for the quantum case is an interesting open question [55,56]. Our findings are immensely useful for aiabatic light transfer $[57,58]$, quantum communication and state transfer in cavitybased quantum networks $[8,59]$ and for nonlinear waveguide optics [46].

Future outlook includes adapting these protocols in different fields where a variety of engineered Hamiltonians are achieved (for, e.g., optomechanics [60-63]). It is interesting to generalize our scheme to higher-dimensional systems [3] and complex geometries [1]. An open fundamental question is connecting out-of-time-ordered correlator $[64,65]$ and LE in our STIRAP setup especially because STIRAP is a unique platform to access both chaotic and nonchaotic regimes.

Acknowledgments. We thank L. Santos, M. A. Bastarrachea-Magnani, D. O’Dell, A. A. Clerk, V. Singh, J. Petta, R. Vijayraghavan, and A. K. Chatterjee for useful discussions. M.K. gratefully acknowledges the Ramanujan Fellowship No. SB/S2/RJN-114/2016 from the Science 
and Engineering Research Board (SERB), Department of Science and Technology, Government of India. M.K. also acknowledges support from the Early Career Research Award No. ECR/2018/002085 from the Science and Engineering Research Board (SERB), Department of Science and Technology, Government of India. M.K. would like to acknowledge support from the Project No. 6004-1 of the Indo-French Centre for the Promotion of Advanced Research (IFCPAR). M.K. acknowledges support from the Matrics Grant, Grant No. MTR/2019/001101 from the Science and Engineering Research Board (SERB), Department of Science and Technology, Government of India.
[1] A. J. Kollár, M. Fitzpatrick, and A. A. Houck, Nature (London) 571, 45 (2019).

[2] M. Fitzpatrick, N. M. Sundaresan, A. C. Y. Li, J. Koch, and A. A. Houck, Phys. Rev. X 7, 011016 (2017).

[3] A. A. Houck, H. E. Türeci, and J. Koch, Nat. Phys. 8, 292 (2012).

[4] S. Schmidt and J. Koch, Anns. Phys. (Berlin) 525, 395 (2013).

[5] J. I. Cirac, P. Zoller, H. J. Kimble, and H. Mabuchi, Phys. Rev. Lett. 78, 3221 (1997).

[6] J. Long, H. S. Ku, X. Wu, X. Gu, R. E. Lake, M. Bal, Y. X. Liu, and D. P. Pappas, Phys. Rev. Lett. 120, 083602 (2018).

[7] B. Vogell, B. Vermersch, T. E. Northup, B. P. Lanyon, and C. A. Muschik, Quant. Sci. Technol. 2, 045003 (2017).

[8] S. Kato, N. Német, K. Senga, S. Mizukami, X. Huang, S. Parkins, and T. Aoki, Nat. Commun. 10, 1160 (2019).

[9] N. Meher and S. Sivakumar, and P. K. Panigrahi, Sci. Rep. 7, 9251 (2017).

[10] A. Biswas and G. S. Agarwal, Phys. Rev. A 70, 022323 (2004).

[11] M. J. Hartmann, F. G. S. Brandão, and M. B. Plenio, Nat. Phys. 2, 849 (2006).

[12] J. J. Mendoza-Arenas, S. R. Clark, S. Felicetti, G. Romero, E. Solano, D. G. Angelakis, and D. Jaksch, Phys. Rev. A 93, 023821 (2016).

[13] R. Coto, M. Orszag, and V. Eremeev, Phys. Rev. A 91, 043841 (2015).

[14] J. Jin, D. Rossini, M. Leib, M. J. Hartmann, and R. Fazio, Phys. Rev. A 90, 023827 (2014).

[15] A. Dey and M. Kulkarni, Phys. Rev. A 101, 043801 (2020).

[16] J. Raftery, D. Sadri, S. Schmidt, H. E. Türeci, and A. A. Houck, Phys. Rev. X 4, 031043 (2014).

[17] S. Schmidt, D. Gerace, A. A. Houck, G. Blatter, and H. E. Türeci,Phys. Rev B 82, 100507(R) (2010).

[18] A. D. Greentree, C. Tahan, J. H. Cole, and L. C. L. Hollenberg, Nat. Phys. 2, 856 (2006).

[19] D. Rossini and R. Fazio, Phys. Rev. Lett. 99, 186401 (2007).

[20] M. Aichhorn, M. Hohenadler, C. Tahan, and P. B. Littlewood, Phys. Rev. Lett. 100, 216401 (2008).

[21] O. T. Brown and M. J. Harmann, New J. Phys. 20, 055004 (2018).

[22] H. J. Kimble, Nature (London) 453, 1023 (2008).

[23] A. Reiserer and G. Rempe, Rev. Mod. Phys. 87, 1379 (2015).

[24] M. Orszag, N. Ciobanu, R. Coto, and V. Eremeev, J. Mod. Opt. 62, 593 (2014).

[25] C. Aron, M. Kulkarni, and H. E. Türeci, Phys. Rev. X 6, 011032 (2016).

[26] A. Dey, D. Cohen, and A. Vardi, Phys. Rev. Lett. 121, 250405 (2018)

[27] E. M. Graefe, H. J. Korsch, and D. Witthaut, Phys. Rev. A 73, 013617 (2006).
[28] A. Dey, D. Cohen, and A. Vardi, Phys. Rev. A 99, 033623 (2019).

[29] J. Larson and D. H. J. O'Dell, J. Phys. B: At. Mol. Opt. Phys. 46, 224015 (2013).

[30] M. A. B.-Magnani, B. L.-del-Carpio, J. C.-Carlos, S. L.-Hernández, and J. G. Hirsch, Phys. Scr. 92, 054003 (2017).

[31] S. V. Prants and V. Y. Sirotkin, Phys. Rev. A 64, 033412 (2001).

[32] S. V. Prants and M. Y. Uleysky, JETP Lett. 82, 748 (2005).

[33] S. V. Prants, JETP Lett. 75, 651 (2002).

[34] S. Felicetti, G. Romero, D. Rossini, R. Fazio, and E. Solano, Phys. Rev. A 89, 013853 (2014).

[35] Y. Lahini, F. Pozzi, M. Sorel, R. Morandotti, D. N Christodoulides, and Y. Silberberg, Phys. Rev. Lett. 101, 193901 (2008).

[36] See Supplemental Material at http://link.aps.org/supplemental/ 10.1103/PhysRevResearch.2.042004 for fully quantum cases and for the results after including the presence of dissipation.

[37] G. D. Vallea, M. Ornigotti, T. T. Fernandez, P. Laporta, and S. Longhi, Appl. Phys. Lett. 92, 011106 (2008).

[38] A. M. Kenis, I. Vorobeichik, M. Orenstein, and N. Moiseyev, IEEE J. Quantum Electron. 37, 1321 (2001).

[39] S. Longhi, G. Della Valle, M. Ornigotti, and P. Laporta, Phys. Rev. B 76, 201101(R) (2007).

[40] E. Paspalakis, Opt. Commun. 258, 31 (2006).

[41] B. T. Torosov, G. Della Valle, and S. Longhi, Phys. Rev. A 89 , 063412 (2014).

[42] L. Tian, M. S. Allman, and R. W. Simmonds, New J. Phys. 10, 115001 (2008).

[43] Y. Chen, C. Neill, P. Roushan, N. Leung, M. Fang, R. Barends, J. Kelly, B. Campbell, Z. Chen, B. Chiaro, A. Dunsworth, E. Jeffrey, A. Megrant, J. Y. Mutus, P. J. J. O'malley, C. M. Quintana, D. Sank, A. Vainsencher, J. Wenner, T. C. White, M. R. Geller, A. N. Cleland, and J. M. Martinis, Phys. Rev. Lett. 113, 220502 (2014).

[44] M. Boissonneault, J. M. Gambetta, and A. Blais, Phys. Rev. A 79, 013819 (2009).

[45] K. Bergmann, H. Theuer, and B. W. Shore, Rev. Mod. Phys. 70 , 1003 (1998).

[46] N. V. Vitanov, A. A. Rangelov, B. W. Shore, and K. Bergmann, Rev. Mod. Phys. 89, 015006 (2017).

[47] K. Bergmann et al., J. Phys. B: At. Mol. Opt. Phys. 52, 202001 (2019).

[48] M. Kulkarni, O. Cotlet, and H. E. Türeci, Phys. Rev. B 90 , 125402 (2014)

[49] M. Casartelli, E. Diana, L. Galgani, and A. Scotti, Phys. Rev A 13, 1921 (1976).

[50] G. Benettin, L. Galgani, A. Giorgilli, and J.-M. Strelcyn, Meccanica 15, 9 (1980). 
[51] G. Benettin, L. Galgani, A. Giorgilli, and J.-M. Strelcyn, Meccanica 15, 21 (1980).

[52] G. Benettin, L. Galgani, and J.-M. Strelcyn, Phys. Rev. A 14, 2338 (1976).

[53] T. A. Elsayed, B. Hess, and B. V. Fine, Phys Rev E 90, 022910 (2014).

[54] T. Mathisen and J. Larson, Entropy 20, 20 (2018).

[55] F. Mattinson, M. Kira, and S. Stenholm, J. Mod. Opt. 48, 889 (2001).

[56] J. Larson and E. Andersson, Phys. Rev. A 71, 053814 (2005).

[57] N. Miladinovic, F. Hasan, N. Chisholm, I. E. Linnington, E. A. Hinds, and D. H. J. O'Dell, Phys. Rev. A 84, 043822 (2011).

[58] F. Hasan and D. H. J. O’Dell, Phys. Rev. A 94, 043823 (2016).
[59] L.-B. Chen, M.-Y. Ye, G.-W. Lin, Q.-H. Du, and X.-M. Lin, Phys. Rev. A 76, 062304 (2007).

[60] Y. D. Wang and A. A. Clerk, Phys. Rev. Lett. 108, 153603 (2012).

[61] M. Aspelmeyer, T. J. Kippenberg, and F. Marquardt, Rev. Mod. Phys. 86, 1391 (2014).

[62] Y.-D. Wang and A. A. Clerk, New. J. Phys. 14, 105010 (2012).

[63] L. Tian, Phys. Rev. Lett. 108, 153604 (2012).

[64] J. Chávez-Carlos, B. López-del-Carpio, M. A. BastarracheaMagnani, P. Stránsky, S. Lerma-Hernández, and L. F. Santos, and J. G. Hirsch, Phys. Rev. Lett. 122, 024101 (2019).

[65] S. Pilatowsky-Cameo, J. Chávez-Carlos, M. A. BastarracheaMagnani, P. Stránsky, S. Lerma-Hernández, and L. F. Santos, and J. G. Hirsch, Phys. Rev. E 101, 010202(R) (2020). 